\title{
Study of induced half-metallic ferromagnetic property in CdO monolayer with non-metallic doping
}

\author{
Lalmuanchhana ${ }^{1}$, Lalrinthara Pachuau ${ }^{2}$, Dibya Prakash Rai ${ }^{2}$, Ramesh Chandra Tiwari ${ }^{1}$, \\ Lalhriatzuala ${ }^{2^{*}}$ \\ ${ }^{1}$ Department of Physics, School of Physical Sciences, Mizoram University, Aizawl 796004 \\ 2Department of Physics, Mizoram University, Pachhunga University College, Aizawl 796001
}

\begin{abstract}
Doping is known to be an efficient way of altering the properties of materials. We have studied the structural, electronic and magnetic properties of non-metallic element ( $\mathrm{B}, \mathrm{C}$ and $\mathrm{N}$ )-doped $\mathrm{CdO}$ monolayer based on spin-polarized density function theory within the generalized gradient approximation (GGA) as revised for solids by Perdew, Burke and Ernzerhof (PBE-sol). Substituting B, C and N in O site with one atom in $\mathrm{CdO}$ monolayer induces spin polarized localized states in the gap or near the valence band and exhibit local magnetic moments $\sim 1 \mu \mathrm{B}, \sim 2 \mu \mathrm{B}$ and $\sim 1 \mu \mathrm{B}$ respectively. It is found that the magnetic states in these doped systems are associated with the difference between the electronegativity of the dopant and the anion in the host. Our study shows that non-metal doped $\mathrm{CdO}$ is a promising candidate for novel nano-materials for future applications.
\end{abstract}

Received 22 May 2021 Accepted 13 Junly 2021

*For correspondence: hriata@pucollege.edu.in

Contact us: sciencevision@outlook.com

Keywords: Doping, CdO monolayer, non-metallic element, spin.

\section{Introduction}

Advancement in the modern technology ignites surge for the miniaturization of electronic devices. Series of 2D nano-materials with unique physical and chemical properties have drawn much attention since graphene was successfully prepared in $2004{ }^{1}$ These 2D materials exhibit intriguing and abundant physical properties, ranging from metallic conductor to semiconductor, which guarantee them promising wide applications in electronic and optoelectronic devices in nano-scale..$^{2-4}$ Many low dimensional materials such as silicone, ${ }^{5,6}$ transition metals dichalcogenide $^{7-9}$ and hexagonal boron nitride ${ }^{10}$ have been successfully prepared in experiments. Theoretical-based DFT calculations are also employed to predict new 2D material, ${ }^{11-17}$ specifically among the honeycomb monolayers of II-VI group which exhibit tuneable properties. ${ }^{18-20}$

Among II-VI group compounds, $\mathrm{CdO}$ single layer is found to exhibit a good dynamic stability using first principles calculations. ${ }^{21}$ With its tuneable properties, this result motivated further studies of the doped $\mathrm{CdO}$ single layer physical properties. But the issue of doping with non-transitional metal is that when electronegativity of the dopant is weaker than the replaced atom, it leads to ferromagnetism and if the electronegativity of the dopant is stronger than the replaced atom, it does not induce spinpolarisation. 22 These results indicate that the electronegativity difference between the dopant and the anion of the host semiconductor plays an 
Table 1 | Structural parameters of pure CdO ML, B-doped CdO ML, C-doped CdO ML and N-doped CdO ML. D represent the dopant atom $(B / C / N)$. Ef is the formation energy of the doped $C d O M L$.

\begin{tabular}{|c|c|c|c|c|c|c|}
\hline \multirow[t]{2}{*}{ System } & \multirow{2}{*}{$\begin{array}{c}\text { Ground state } \\
\text { energy (eV) }\end{array}$} & \multicolumn{2}{|c|}{ Bond length $(\AA)$} & \multicolumn{2}{|c|}{ Bond angle ( $\left.{ }^{\circ}\right)$} & \multirow{2}{*}{$\begin{array}{c}E_{f} \\
(e V)\end{array}$} \\
\hline & & Cd-O & Cd-D & $\theta_{c d-0-c d}$ & $\theta_{C d-D-C d}$ & \\
\hline Pure CdO ML & -121.970603 & $\begin{array}{l}2.027 \\
2.07 *\end{array}$ & - & 120 & - & - \\
\hline B-doped CdO ML & -117.486384 & $\begin{array}{l}2.024 \\
2.06 *\end{array}$ & $\begin{array}{c}2.09 \\
2.10^{*}\end{array}$ & 121.88 & 120.01 & $\begin{array}{l}+5.116 \\
6.68 *\end{array}$ \\
\hline C-doped CdO ML & -119.682749 & $\begin{array}{l}2.024 \\
2.07 *\end{array}$ & $\begin{array}{c}2.03 \\
2.07 *\end{array}$ & 122.17 & 119.99 & $\begin{array}{l}2.959 \\
7.25 *\end{array}$ \\
\hline N-doped CdO ML & -120.969983 & $\begin{array}{l}2.024 \\
2.07 *\end{array}$ & $\begin{array}{c}2.03 \\
2.06 *\end{array}$ & 121.29 & 119.99 & $\begin{array}{l}+1.67 \\
4.76 *\end{array}$ \\
\hline
\end{tabular}

* Results obtained by Chaurasiya et al. [36] using GGA-PBE.

Table 2: Band gap of pure CdO ML, B-doped CdO ML, C-doped CdO ML and N-doped CdO ML, local magnetic moments of dopants $(\mathrm{B} / \mathrm{C} / \mathrm{N})$ and magnetic moments per supercell of the doped structures.

\begin{tabular}{lccc|} 
System & Band gap (eV) & $\begin{array}{c}\text { Local magnetic moment } \\
\text { of dopant }(\mu \mathrm{B})\end{array}$ & $\begin{array}{c}\text { Magnetic moment per } \\
\text { supercell }(\boldsymbol{\mu})\end{array}$ \\
\hline Pristine CdO & 0.69 & & 0.99 \\
\hline B-doped CdO & 0.35 & 0.115 & $0.98^{*}$ \\
\hline C-doped CdO & 0.64 & $0.36^{*}$ & 1.99 \\
& & 0.497 & $1.99^{*}$ \\
\hline N-doped CdO & 0.69 & $1.32^{*}$ & 0.99 \\
& & 0.434 & $0.99^{*}$ \\
\hline
\end{tabular}

* Results obtained by Chaurasiya et al. [36] using GGA-PBE.

important role for the magnetism of such doped semiconductors. However, the tuneable properties of these materials are capable of enhancing the electrical, optical, and structural properties of a semiconductor and open a new way in the advancement of miniature technology.

Cadmium oxide $(\mathrm{CdO})$ is an n-type oxide belonging to the $\mathrm{II}-\mathrm{VI}$ group having direct and indirect band gaps in the range of 2.2-2.5 eV and $1.36-1.98 \mathrm{eV}$ respectively. ${ }^{23-25}$ Due to its ionic nature and low electrical resistivity and high optical transmission in the visible region, nano-scaled $\mathrm{CdO}$ are suitable candidate for several applications in various fields such as optical coatings, photovoltaic cells, phototransistors, IR reflectors, transparent electrodes, and gas sensor. ${ }^{26-33}$ Various $\mathrm{CdO}$ nanostructures have been, in fact, studied in recent years. ${ }^{34-40}$ Zheng et al. ${ }^{21}$ confirmed the structural stability using density functional theory (DFT), Khan et $a l^{40}$ studied the electronic and optical properties of $\mathrm{CdO}$ bulk and monolayer and provided a relative comparison. Xiaoxuan et al. ${ }^{38}$ explored electronic properties of $\mathrm{CdO}$ monolayer and nanotube. The strain effect on the optoelectronic properties of $\mathrm{CdO}$ monolayer has been investigated by Chaurasiya et $a{ }^{34}$ and results indicate that the band gap decreases when the lattice distortion is applied and the directindirect gap transition can be achieved at tensile strain of $8 \%$. Chaurasiya et al. ${ }^{36}$ also presented the magnetism induced upon point defects and doping with non-transitional metal with $\mathrm{CdO}$ monolayer.

The approximation considered to the correlationexchange functional in our DFT calculation is the generalized gradient approximation (GGA) in the form of Perdew, Burke and Ernzerhof for solids (PBEsol) ${ }^{41}$ Although popular Generalized Gradient Approximation (GGA) ${ }^{42}$ is widely used, it is biased on account of free atom energies. ${ }^{41}$ PBE-sol is the restoration of the first principles gradient expansion for exchange over a wide range of density gradients. GGA (PBE-sol) is found to be more appropriate than GGA (PBE) for structural properties in solids and are 
(a)

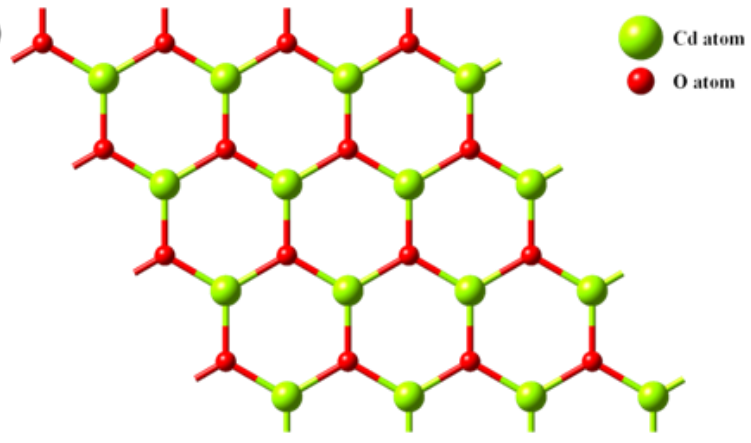

(b)

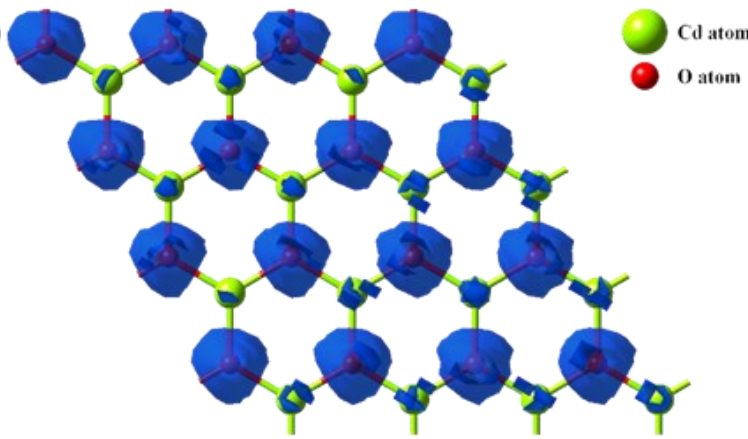

Figure 1 | (a) Schematic diagram of 4x4 CdO monolayer. (b) ELF of pure CdO monolayer.

in good agreement with experimental values. ${ }^{43}$ The GGA (PBE-sol) thus eliminates the biasing towards the description of free atom energies and improved equilibrium properties of densely packed solids and their surfaces. ${ }^{41,44}$

In this work, we present a comprehensive and comparative study of the structural, electronic and magnetic properties of $\mathrm{B}, \mathrm{C}$ and $\mathrm{N}$ doped $\mathrm{CdO} \mathrm{ML}$ obtained using spin-polarized density functional theory calculations. The structural stability of the monolayer is confirmed by the ground state energy. It is observed that the doped structures exhibit halfmetallic behaviour due to the spin polarized $2 p$ orbitals of the dopant, which are localized within the energy gap of the host lattice. The analysis of electronic structures showed that the d-orbitals of Cd play a crucial role in the electronic characteristics such as defining its valence band maxima and accommodation of most charges of its host atom.

\section{Computational Details}

In this study, a $4 \times 4$ supercell of $\mathrm{CdO}$ monolayer with 16 atoms each of $\mathrm{Cd}$ and $\mathrm{O}$ is taken as the undoped structure. A vacuum of $15 \AA$ is inserted along the $z$-direction to avoid the interactions between the monolayers during periodic calculations. Carbon, boron and nitrogen doping are done in the structure by substituting one of the oxygen atoms with a single dopant atom, thus making a doped structure with one dopant $(B / C / N)$ atom in every 15 oxygen atoms.

The doped structures are optimized until the forces on each atom have become less than $0.02 \mathrm{eV} /$ $\AA$ and by setting the energy convergence criteria of $10^{-5} \mathrm{eV}$. All the computations are performed within the framework of spin-polarized plane-wave density functional theory (DFT) as implemented in the Vienna Ab-initio simulation package (VASP). ${ }^{45}$ The ionic potentials are represented by projector augmented wave potentials (PAW) ${ }^{46}$ and electron exchange correlation is represented by generalized gradient approximation (GGA) as revised for solids by Perdew, Burke and Ernzerhof (PBE-sol). ${ }^{41}$ An energy cut-off of $400 \mathrm{eV}$ is used for the plane-wave expansion of the electronic wave function and the Brillouin zone integration is performed using $8 \times 8 \times 1$ k-point mesh within the Monkhorst-Pack scheme. ${ }^{47}$ For electronic structure calculations, the Brillouin zone is sampled by $16 \times 16 \times 1 \mathrm{k}$-point mesh.

\section{Results and Discussion}

The schematic diagram for top view of a $4 \times 4 \mathrm{CdO}$ monolayer is shown in Fig 1(a). The lattice parameter of pristine $\mathrm{CdO}$ monolayer is optimized by calculating the total energy of monolayer. The total energy of CdO ML after structural optimization is $121.97 \mathrm{eV}$ indicating that the structure is stable. The optimized bond length between $\mathrm{Cd}$ and $\mathrm{O}$ is $2.03 \AA$. $\mathrm{Cd}$ and $\mathrm{O}$ atoms lie in $\mathrm{X}-\mathrm{Y}$ plane, forming a graphene like honeycomb structure, suggesting sp2 hybridization. The $\mathrm{Cd}-\mathrm{O}-\mathrm{Cd} / \mathrm{O}-\mathrm{Cd}-\mathrm{O}$ bond angle is $120^{\circ}$, showing no buckling height suggesting a planar stability. The calculated values are comparable with reported literatures. ${ }^{21,36,38}$

The electronic properties of $\mathrm{CdO}$ monolayer are investigated by computing the band structure and density of states. The band structure is calculated using spin polarized GGA exchange-correlation functional along $\Gamma-\mathrm{M}-\mathrm{K}-\Gamma$ Brillouin zone. The Fermi energy is set at $0 \mathrm{eV}$, as shown in Fig 2(a). The band structure showed that valance band maxima and conduction band minima are located at $\Gamma$ point indicating a direct bandgap nature with a band gap value of about $0.691 \mathrm{eV}$. The computed bandgap value is in agreement with $0.66 \mathrm{eV}$ as reported by Zheng et al. ${ }^{21}$ However, the computed value is much lower than the experimentally reported value of 3.3 eV for thin films. ${ }^{33}$ This difference may be attributed to GGA exchange-correlation functions used for present calculations, which underestimates the bandgap of semiconducting materials. The calculated total and partial density of states are shown in Fig 2(b). The electronic energy states near Fermi energy in the valence band are contributed by cadmium $4 d$ orbitals and oxygen $2 p$ orbitals while cadmium $5 \mathrm{~s}$, cadmium $4 \mathrm{p}$ and oxygen $2 \mathrm{~s}$ orbitals are 
(a)

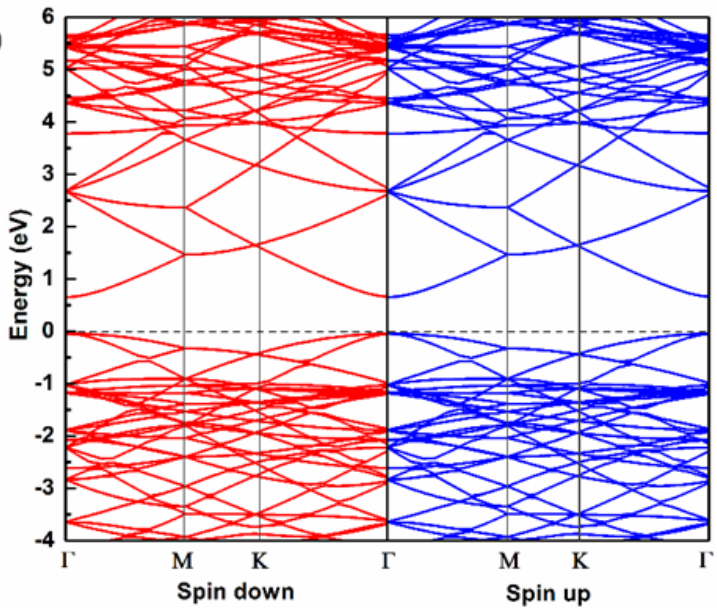

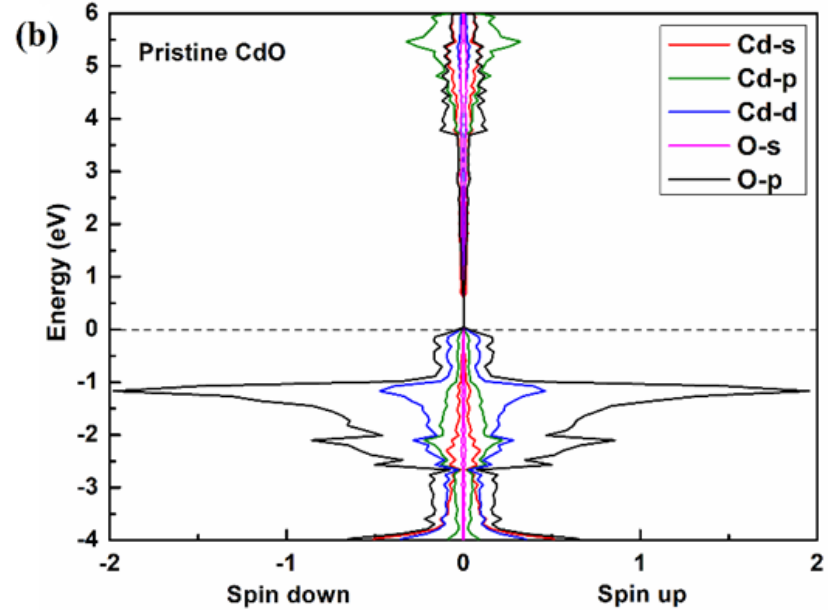

Figure 2 | (a) Bandstructure of pure CdO ML. (b) PDOS of pure CdO ML.

(a)
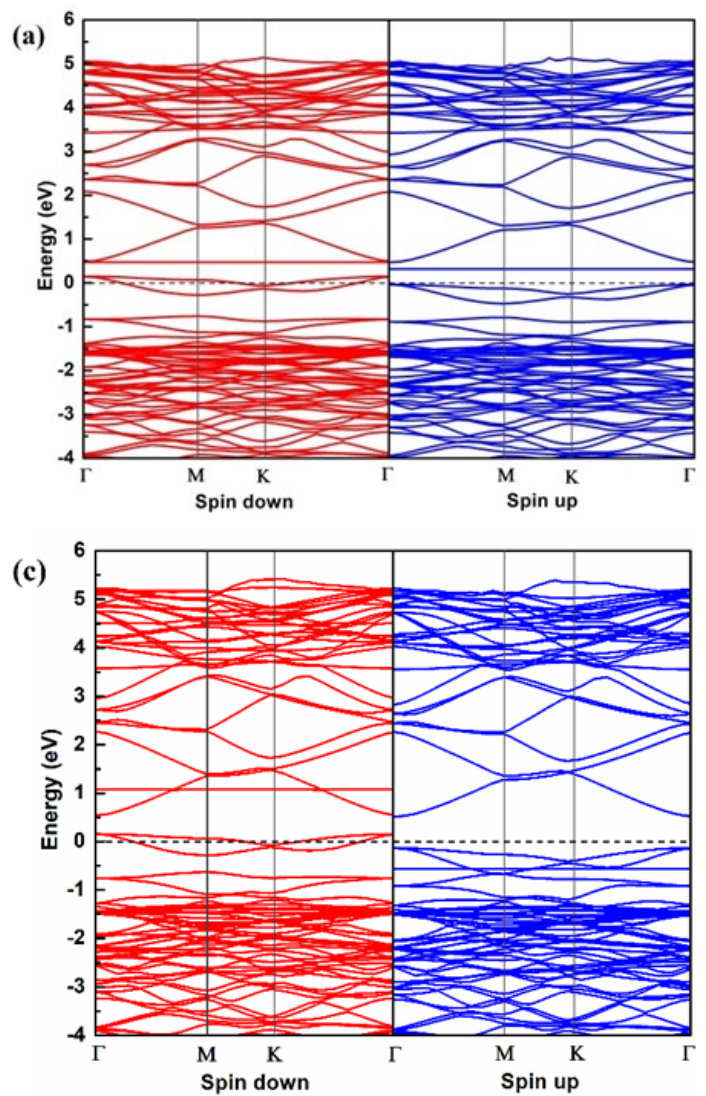

(e)

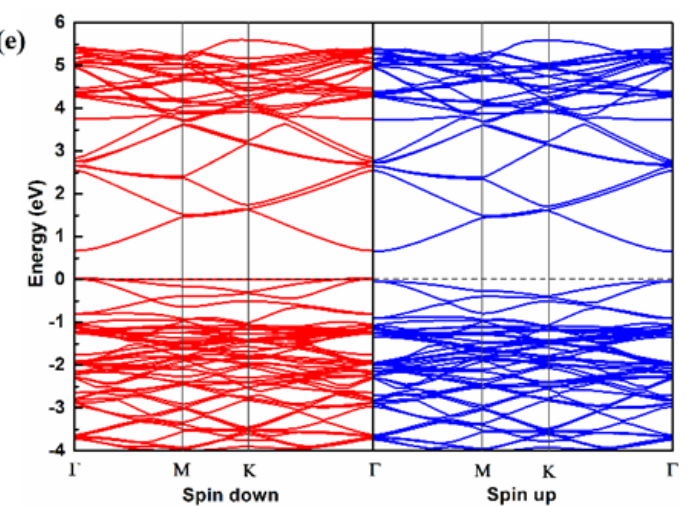

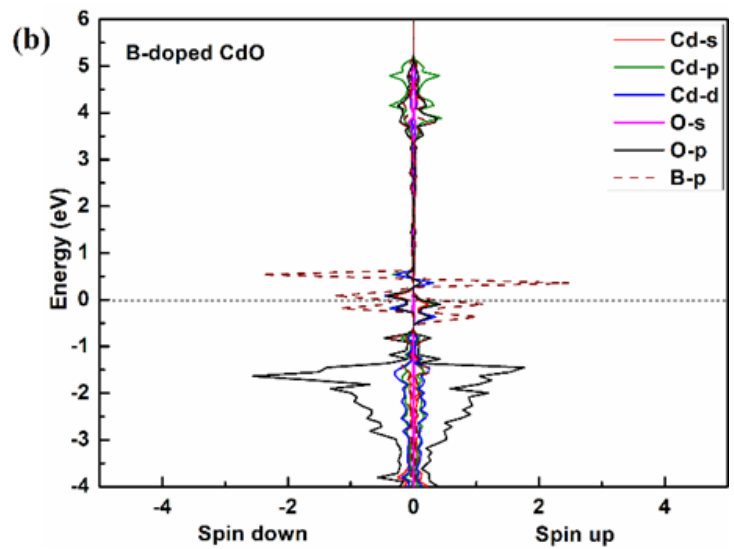
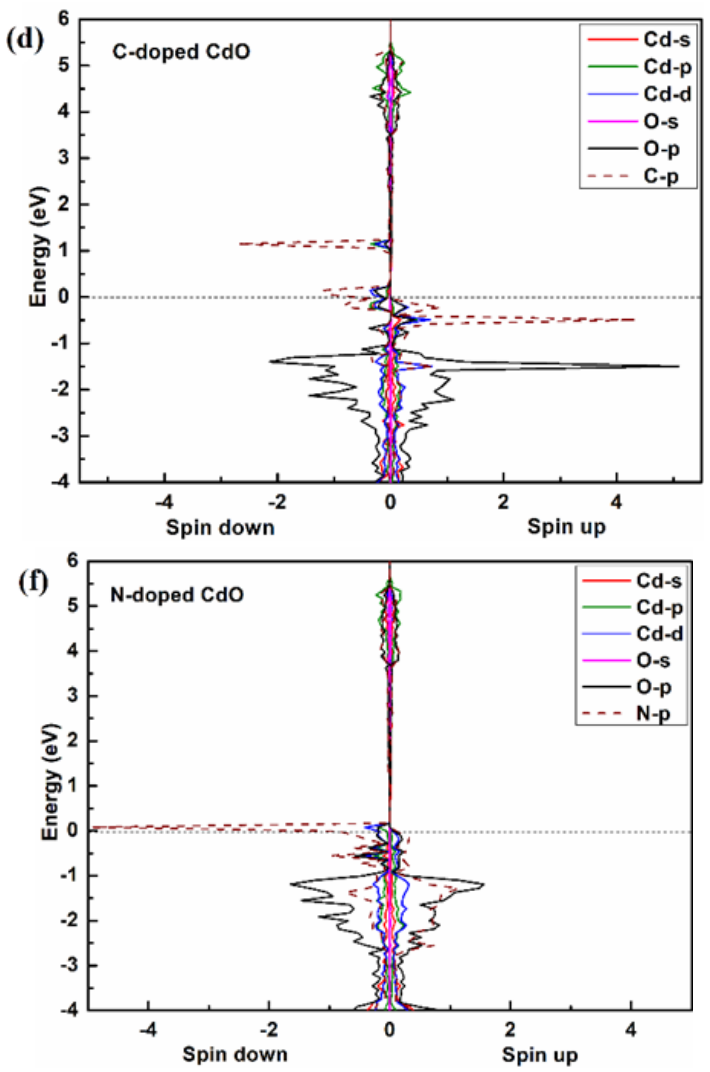

Figure 3 | (a) Bandstructure of B-doped CdO ML. (b) PDOS of B-doped CdO ML. (c) Bandstructure of C-doped CdO ML. (d) PDOS of C-doped CdO ML. (e) Bandstructure of N-doped CdO ML (f) PDOS of N-doped CdO ML. 
(a)

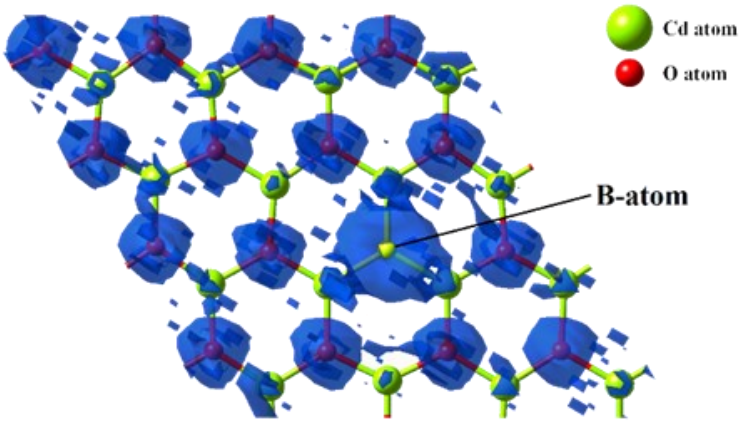

(b)

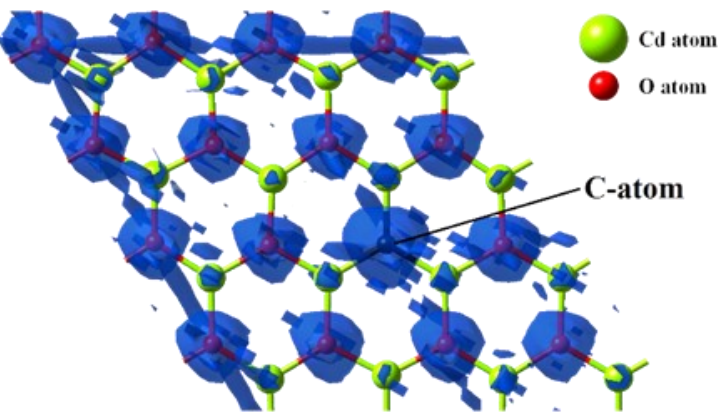

(c)

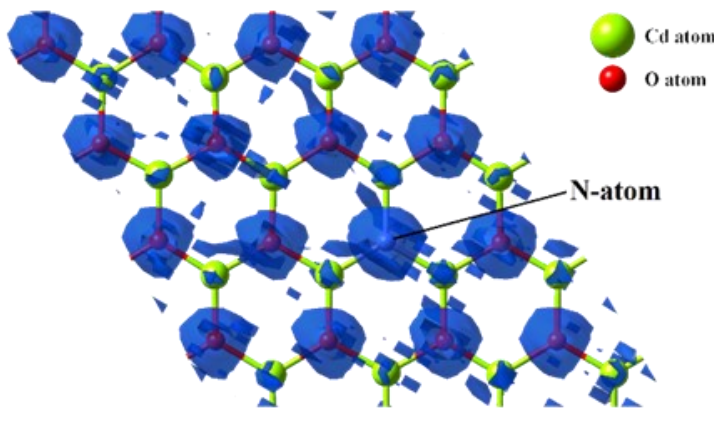

Figure 4 | ELF of (a) B-doped CdO ML; (b) C-doped CdO ML; (c) N-doped CdO ML.

(a)

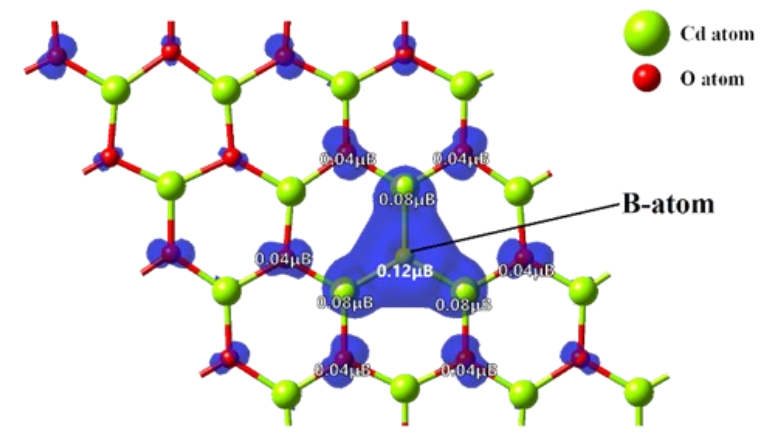

(b)

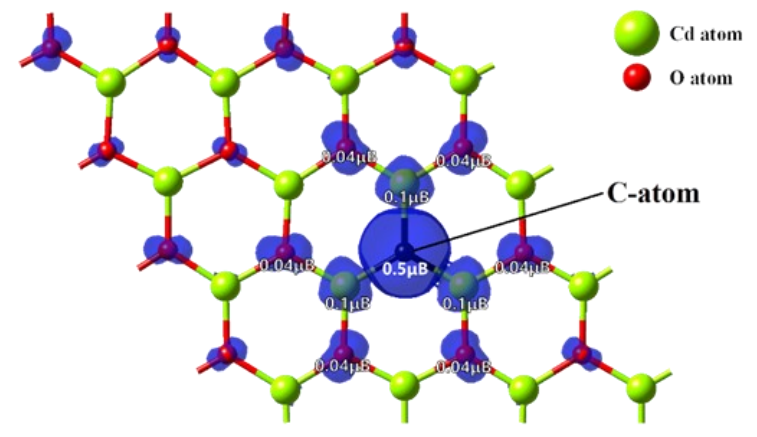

(c)

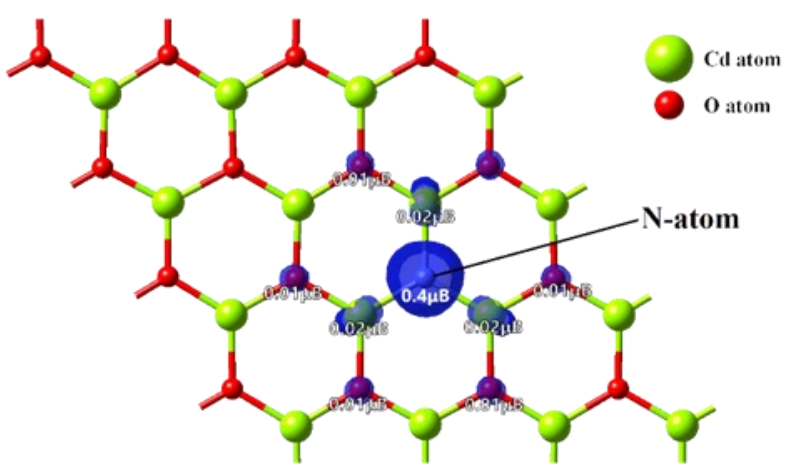

Figure 5 | Magnetization density of (a) B-doped CdO ML, (b) C-doped CdO ML, (c) N-doped CdO ML 
contributing to the deeper valence bands. The conduction band minima are mainly contributed by cadmium $5 \mathrm{~s}$ orbitals. The symmetrical density of states (DOS) for spin up and spin down states confirm the non-magnetic behaviour of pristine $\mathrm{CdO}$ monolayer.

The atomic charge density is calculated to understand the charge sharing and bonding among atoms. The DFT calculations showed that oxygen atoms act as a charge accumulating site while cadmium atoms behave like charge depleting sites. The transfer of charge from $\mathrm{Cd}$ atoms to $\mathrm{O}$ atoms is confirmed from Mulliken charge analysis, where $1.2 \mathrm{e}$ excess charge each is observed on oxygen atoms. Electron localization function (ELF) plot in figure $1(b)$ shows that the electronic charges are more localized around the $\mathrm{O}$ atoms and therefore the chemical bonding in $\mathrm{CdO}$ monolayer is mostly ionic in nature. Atomic partial charges are also computed which shows that most of the charges, i.e. 9.757 e out of the total 10.828 e, are located on the $d$ orbitals of $\mathrm{Cd}$ atoms. While in $\mathrm{O}$ atoms, majority of the charges are on $p$ orbitals, i.e., 2.803e out of 4.057e total charges and there is also a significant amount of charges on the $s$ orbitals, i.e., $1.249 \mathrm{e}$ out of $4.057 \mathrm{e}$ total charges. This indicates that the filled valence bands near Fermi level are mostly the bonding states of $4 \mathrm{~d}$ orbitals of $\mathrm{Cd}$ atoms and $2 \mathrm{p}$ orbitals of $\mathrm{O}$ atoms.

For doping, one of the $\mathrm{O}$ atoms in the $4 \times 4 \mathrm{CdO}$ supercell is substituted by the dopant atom. By the same parameters used in the pure $\mathrm{CdO}$ system, the doped structure is then optimized using spin polarized DFT calculations. All the doped structures are perfectly planar without any buckling. However, due to the insertion of smaller atoms at the $O$ site, the bond length and bond angle slightly change around the dopant atom. The bond length between $\mathrm{Cd}$ and $\mathrm{B}$ atoms is $2.09 \AA$ and the bond angle around the $\mathrm{B}$ atom, i.e., $\mathrm{Cd}-\mathrm{B}-\mathrm{Cd}$ is $120.01^{\circ}$. For $\mathrm{C}$ doped sample, bond length between $\mathrm{Cd}$ and $\mathrm{C}$ atoms is $2.03 \AA$ and the bond angle $\mathrm{Cd}-\mathrm{C}-\mathrm{Cd}$ is $119.99^{\circ}$. While for $\mathrm{N}$ doped sample, bond length between $\mathrm{Cd}$ and $\mathrm{N}$ atoms is $2.03 \AA$ and the bond angle $\mathrm{Cd}-\mathrm{N}-\mathrm{Cd}$ is $119.99^{\circ}$. Total energy of the $B, C$ and $N$ doped structures are -117.49 eV,-119.68 eV and -120.96 eV respectively. These negative ground state energies indicate that these doped structures are stable even though $\mathrm{O}$ atom is substituted with dopant atom (B/ $\mathrm{C} / \mathrm{N})$, which possesses smaller ionic radius and resulted in altering of its bond length and bond angle.

Formation energy of the doped structures is calculated to understand their relative stability using the relation:

$$
\begin{aligned}
E_{f}= & E_{\text {total }}(B / C / N \text { doped } C d O)-E_{\text {tot }}(\text { pristine } C d O) \\
& +E_{O}-E_{\text {dopant }}
\end{aligned}
$$

where $E_{\text {total }}(B / C / N$ doped $C d O)$ and $E_{\text {tot }}$ (pristine $\left.C d O\right)$ are the total energies per supercell of the relaxed B/ $\mathrm{C} / \mathrm{N}$ doped and pure $\mathrm{CdO} M \mathrm{ML}$ respectively. $E_{O}$ and $E_{\text {dopant }}$ represent the chemical potential of $O$ and the dopant atoms (B / C/ N) respectively. Formation energies of the $\mathrm{B}$-doped and $\mathrm{C}$-doped $\mathrm{CdO} \mathrm{ML}$ structures are $+5.116 \mathrm{eV}$ and $2.959 \mathrm{eV}$ respectively while $\mathrm{N}$-doped $\mathrm{CdO} \mathrm{ML}$ has $+1.67 \mathrm{eV}$ of formation energy. This indicates that $\mathrm{N}$ doped $\mathrm{CdO} \mathrm{ML}$ is the most favourable stable structure.

The calculated electronic band structure of the B, $\mathrm{C}$ and $\mathrm{N}$ doped $\mathrm{CdO} \mathrm{ML}$ are given in figure $3(\mathrm{a}), 3(\mathrm{c})$ and $3(\mathrm{e})$ respectively. All the doped structures exhibit half-metallic behaviour with one of its spin states (spin down) showing metallic behaviour while the other spin state behaves as a semiconductor. The Fermi level also lies within the valence band for the spin down channel indicating the presence of unoccupied energy states within the valence band. In $\mathrm{B}$-doped $\mathrm{CdO} \mathrm{ML}$, the energy gap in the spin up channel is $0.35 \mathrm{eV}$, which is about $49 \%$ smaller than the pristine $\mathrm{CdO}$ ML direct gap $(0.691 \mathrm{eV})$. B atom having significantly smaller atomic radius occupy $O$ site in the lattice, the chemical bonds with the neighbouring $\mathrm{Cd}$ atoms become stretched and creates a large distortion, which deforms the crystal structure around the dopant atom. This influences the electronic bandstructure and it results in reduced band gap of the host lattice in agreement with reported studies on the effect of strain on $\mathrm{CdO} \mathrm{ML}^{34}$ The C-doped CdO monolayer on the other hand shows energy gap of $0.64 \mathrm{eV}$ which remains almost the same with the pristine structure. The separation between the valence band maximum and conduction band minimum for $\mathrm{N}$-doped $\mathrm{CdO} \mathrm{ML}$ is around $0.69 \mathrm{eV}$ at the $\Gamma$ point. Among the dopants, $\mathrm{B}$ atom significantly modifies the band gap while $C$ and $\mathrm{N}$ dopants offer almost no change as compared to the pristine CdO ML. The flat band line observed in the bandstructure of these doped MLs could be due to the formation of defect energy states by the dopant atom. The two defect states in the case of Bdoped CdO ML are noticed at $0.35 \mathrm{eV}$ and $0.51 \mathrm{eV}$ in the forbidden region near conduction band while in the case of $\mathrm{C}$-doping, the defect states are observed around $-0.58 \mathrm{eV}$ in forbidden region near the valence band. In the case of $\mathrm{N}$-doped $\mathrm{CdO} \mathrm{ML}$, the defect states are observed slightly above the Fermi level in the conduction band. The half-metallicity and observation of defect states is similar to the work of Chaurasiya et $a l^{36}$

Partial density of states (PDOS) corresponding to the doped CdO ML bandstructure are also calculated to understand the atomic contribution in band structure. In B-doped CdO ML system, Fig 3(b), the valence band of the semiconducting spin up channel is contributed by $C d-5 s, C d-4 p, C d-4 d, O-2 p$ and B$2 p$ orbitals. The flat band line observed in the bandstructure, as seen in Fig 3(a), arises due to the presence of $B-2 p, C d-4 d, C d-4 p$, and $0-2 p$ orbitals. The PDOS in Fig 3(b), show that the spin down states 
behave as a metal due to the presence of $B-2 p$ orbital crossing Fermi level. The PDOS of C-doped $\mathrm{CdO} \mathrm{ML}$ given in Fig $3(\mathrm{~d})$, shows that the valence band of the spin up channel is mainly formed by the bonding states of $\mathrm{Cd}-4 \mathrm{~d}, \mathrm{O}-2 \mathrm{p}$ and $\mathrm{C}-2 \mathrm{p}$ orbitals. The plotted PDOS revealed that metallic behaviour for spin down state arises due to $\mathrm{C}-2 \mathrm{p}$ orbitals. Fig 3 (f) shows the calculated PDOS for $\mathrm{N}$-doped CdO ML. The metallic behaviour in the spin down channel is mainly due to the energy states contributed from the $\mathrm{N}-2 \mathrm{p}$ orbitals and in addition to this, there are also contributions from $C d-4 p, C d-4 d$, and $0-2 p$ orbitals. Meanwhile the valence band of the spin up channel can be attributed to $\mathrm{Cd}-4 d, \mathrm{O}-2 \mathrm{p}$ and $\mathrm{N}-2 \mathrm{p}$ orbitals.

Electron Localization Function (ELF) of B-doped, C -doped and $\mathrm{N}$-doped $\mathrm{CdO} \mathrm{ML}$ are given in Fig 4(a), (b) and (c) respectively. In C-doped and N-doped structures, the electronic charges are more localized around the $\mathrm{O}$ atoms and the dopant $\mathrm{C}$ and $\mathrm{N}$-atom in the doped monolayer. This charge localization is similar to the pure $\mathrm{CdO} \mathrm{ML}$ and therefore the bonding in the case of $\mathrm{C}$-doped and $\mathrm{N}$-doped $\mathrm{CdO}$ $\mathrm{ML}$ remains ionic. Bader charge analysis also shows the charge transfer between the atoms. In C-doped $\mathrm{CdO} \mathrm{ML}$, the charge transfer in $\mathrm{C}$ atom is $-0.6287 \mathrm{e}$, and +0.895 e to each of the neighbouring three $\mathrm{Cd}$ atoms. Meanwhile in $\mathrm{N}$-doped structure, the charge transfer in $\mathrm{N}$ atom is -1.0048e and in the neighbouring three $\mathrm{Cd}$ atoms, it is +1.1097 e each. The charge transfer in $\mathrm{C}$ atom is slightly less than that in $\mathrm{O}$ atom meanwhile the charge transfer in $\mathrm{N}$ is roughly the same as that of $\mathrm{O}$ atom. This is due to the lower electron affinity of the $C$ atom in comparison to the $\mathrm{O}$ and $\mathrm{N}$ atoms. In the case of $\mathrm{B}$ doped sample, the charge transfer on the $B$ atom is only $-0.1011 \mathrm{e}$, and +0.7489 e to each of its adjacent $\mathrm{Cd}$ atoms. The electronic charges are also localized around the $B$ atom but the localization is much weaker than around the $\mathrm{O}$ atoms. Electronic charges around the $B$ atom are localized almost near the middle of the chemical bonds with the adjacent three $\mathrm{Cd}$ atoms. Therefore, the chemical bond between $\mathrm{Zn}$ and $\mathrm{B}$ atoms is more of a covalent in nature.

From the spin-polarised calculation, pristine $\mathrm{CdO}$ $\mathrm{ML}$ resulted in zero magnetic moment in all the $s, p$ and $d$ orbitals which shows a non-magnetic behaviour. This is an indication that there are no half -filled atomic orbitals that could result in unpaired electrons responsible for magnetic moment. However, spin-polarized calculations on doped $\mathrm{CdO}$ $\mathrm{ML}$ show the presence of magnetic moments in the structures. The total magnetic moment of $B$ doped CdO monolayer is $0.99 \mu_{\mathrm{B}} \sim 1 \mu_{\mathrm{B}}$ and local magnetic moment of $B$ atom is $0.115 \mu_{B} \sim 0.12 \mu_{B}$. In C-doped system, the total magnetic moment is $1.99 \mu_{B} \sim 2 \mu_{B}$ and local magnetic moment of $C$ atom is $0.497 \mu_{B} \sim$ $0.5 \mu_{\mathrm{B}}$. N-doped $\mathrm{CdO} \mathrm{ML}$ shows a net magnetic moment of $0.991 \mu_{B} \sim 1 \mu_{B}$ and $N$ atom local magnetic moment is $0.4341 \mu_{\mathrm{B}} \sim 0.4 \mu_{\mathrm{B}}$. In the doped system, the magnetic moment arises mainly from the dopant atom and there are also small contributions from the neighbouring $\mathrm{Cd}$ and $\mathrm{O}$ atoms. Whatever magnetic moment observed in $\mathrm{Cd}$ and $\mathrm{O}$ atoms are therefore induced by the dopant atom $(B / C / N)$, which decays gradually as we move further away from it. This variation is also observed in the case of charge transfer from Mulliken charge analysis. In all of the doped structures, the algebraic sum of all the magnetic moments associated with all the atoms in the system equals the net magnetic moment. Thus, it appears that all the magnetic moments associated with the dopant and the host atoms aligned along the same direction, which can happen only if there is a ferromagnetic coupling between the dopant atom $(\mathrm{B} / \mathrm{C} / \mathrm{N})$ and the neighbouring $\mathrm{Cd}$ and $\mathrm{O}$ atoms.

The calculated spin polarized magnetization density obtained for the doped structures are shown in Fig. 6. In both the structures magnetization density is mainly localized on the dopant $B / C / N$ atom and distributed slightly over the three nearest neighbouring $\mathrm{Cd}$ atoms and the next nearest neighbouring $\mathrm{O}$ atoms. Atomic partial magnetic moments are also calculated, which indicates that the magnetic moment in the dopant atom $(B / C / N)$ is mainly due to the $p$ orbitals. In $B$-doped system, the p-orbitals of B-atom contribute $0.110 \mu_{B}$ out of the total $0.115 \mu_{B}$ while the corresponding p-orbitals in $\mathrm{N}$ atom contributes $0.418 \mu_{\mathrm{B}}$ out of the total $0.434 \mu_{\mathrm{B}}$ magnetic moments in their respective structures. In the $\mathrm{C}$-doped system, p-orbitals of $\mathrm{C}$ contribute 0.474 $\mu_{B}$ out of the total $0.497 \mu_{B}$ local magnetic moment associated with the $C$ atom. Fig 5(a) shows overlapping between magnetization densities from $B$ -atom and the three neighboring $\mathrm{Cd}$ atoms in $\mathrm{B}$ doped $M L$, while it is absent in the case of $C$-doped and $\mathrm{N}$-doped structures. This may be attributed to the fact that $\mathrm{B}$-doped $\mathrm{CdO} \mathrm{ML}$ has shorter bond length between $\mathrm{B}$ and its neighboring $\mathrm{Cd}$ atoms. In all the doped structures, atomic partial magnetic moment in the neighbouring three $\mathrm{Cd}$ atoms is largest in the d-orbitals although there are small contributions from the $s$ and $p$ orbitals. The secondary neighbouring $\mathrm{O}$ atoms have its magnetic moment mainly from the $p$ orbitals. This calculation is in agreement with the PDOS of the doped structures in which partially filled B-2p, C-2p, N-2p, $\mathrm{Cd}-4 \mathrm{~d}$ and $\mathrm{O}-2 \mathrm{p}$ orbitals result in metallic behaviour in the spin down channel. Therefore, substitution of $\mathrm{O}$ atom with non-metallic dopant atom $(\mathrm{B} / \mathrm{C} / \mathrm{N})$ having smaller atomic radius and weaker electron affinity results in formation of unpaired electrons transforming semiconducting $\mathrm{CdO} \mathrm{ML}$ into halfmetallic ferromagnets.

\section{Conclusion}

We have studied the impact of doping in $\mathrm{CdO}$ monolayer using spin-polarised density functional 
theory calculations. Pure $\mathrm{CdO}$ monolayer is a thermodynamically stable direct bandgap $(\sim 0.7 \mathrm{eV})$ oxide monolayer system. Our calculations show that doping of $\mathrm{CdO} M L$ with $2 p$ light non-metallic elements such as $\mathrm{B}, \mathrm{C}$, and $\mathrm{N}$ atoms can transform a wide band gap semiconducting $\mathrm{CdO} \mathrm{ML}$ into a halfmetallic ferromagnets. All the dopants; $\mathrm{B}, \mathrm{C}$ and $\mathrm{N}$ atoms being an atom with weaker electronegativity, the charge transfer from the neighbouring $\mathrm{Cd}$ atoms are lesser and resulted in partially filled $2 p$ orbitals of the dopant atom and 4 dorbitals of the neighbouring $\mathrm{Cd}$ atoms, which subsequently influence the secondary neighbouring $\mathrm{O}$ atoms. This results in spin polarized localization of the dopant $2 p$ orbitals and $4 \mathrm{~d}$ orbitals of the neighbouring $\mathrm{Cd}$ atoms as well as $2 p$ orbitals of the subsequent neighbouring $O$ atoms within the energy gap of the host $\mathrm{CdO} \mathrm{ML}$, which is responsible for the half-metallic ferromagnetic behaviour. Among the doped system, C-doped CdO $\mathrm{ML}$ appears to be most favourable while $\mathrm{N}$-doped system appears to be least stable. Atomic partial magnetic moment calculations show that the magnetic property of the doped $\mathrm{CdO} M \mathrm{ML}$ is mainly due to the partially filled $2 \mathrm{p}$ orbitals of the dopant atom $(B / C / N)$. Ferromagnetic coupling is also observed between the dopant atom and the three neighbouring $\mathrm{Cd}$ atoms and the subsequent secondary neighbouring $\mathrm{O}$ atoms.

Comparison of our results with the results obtained by Chaurasiya et al. ${ }^{36}$ on this particular study shows there are noticeable difference in its properties especially its structural parameters of the proposed ML. These differences may be attributed to the approximation considered to the correlationexchange functionals for the study. The previous work done by Chaurasiya et al. ${ }^{36}$ is based on GGA (PBE) whereas our calculation is based upon GGA (PBE-sol). GGA (PBE-sol), which is the restoration of the first principles gradient expansion for exchange over a wide range of density gradients, is found to be more appropriate than GGA (PBE) for structural properties in solids and are in fact in good agreement with experimental values. ${ }^{43}$ Nonetheless, these studies shows that doping of $\mathrm{CdO} \mathrm{ML}$ with non-metal is a promising way to tune its electronic and magnetic properties making it a suitable 2D system for numerous future nano-electronics and spintronic devices.

\section{References}

1. Novoselov, K.S., et al. (2004). Electric Field Effect in Atomically Thin Carbon Films, Science, 306 (5696), 666.

2. Mak, K.F., Shan, J., (2016). Photonics and optoelectronics of 2D semiconductor transition metal dichalcogenides. Nature Photonics, 10(4), 216 -226 .
3. Bonaccorso, F., et al. (2010). Graphene photonics and optoelectronics. Nature Photonics, 2010. 4(9), 611-622.

4. Bao, Q., Loh., K.P. (2012). Graphene Photonics, Plasmonics, and Broadband Optoelectronic Devices. ACS Nano, 6(5), 3677-3694.

5. Vogt, P., et al. (2012). Silicene: Compelling Experimental Evidence for Graphene-like TwoDimensional Silicon. Physical Review Letters, 108 (15), 155501.

6. Tao, L., et al. (2015). Silicene field-effect transistors operating at room temperature. Nature Nanotechnology, 10(3), 227-231.

7. Cong, C., et al. (2014). Synthesis and Optical Properties of Large-Area Single-Crystalline 2D Semiconductor WS2 Monolayer from Chemical Vapor Deposition. Advanced Optical Materials, 2(2), 131-136.

8. Lan, C., et al. (2105). Correction: Large-area synthesis of monolayer $\mathrm{WS}_{2}$ and its ambientsensitive photo-detecting performance. Nanoscale, 7(42), 17980.

9. Xu, S., Li, D., Wu, P., (2015). One-Pot, Facile, and Versatile Synthesis of Monolayer MoS2/WS2 Quantum Dots as Bio-imaging Probes and Efficient Electrocatalysts for Hydrogen Evolution Reaction. Advanced Functional Materials, 25(7), 1127 -1136 .

10. Kim, K.K., et al. (2012). Synthesis of Monolayer Hexagonal Boron Nitride on $\mathrm{Cu}$ Foil Using Chemical Vapor Deposition. Nano Letters, 12(1), 161-166.

11. Hoat, D.M., et al. (2020). Reducing the electronic band gap of $\mathrm{BN}$ monolayer by coexistence of $\mathrm{P}(\mathrm{As})$ -doping and external electric field. Superlattices and Microstructures, 137, 106357.

12. Obeid, M.M., et al. (2019). Electronic and magnetic properties of single-layer boron phosphide associated with materials processing defects. Computational Materials Science, 170, 109201.

13. Hoat, D.M., et al. (2019). Assessing optoelectronic properties of $\mathrm{PbI} 2$ monolayer under uniaxial strain from first principles calculations. Superlattices and Microstructures, 130, 354-360.

14. Nguyen, H.T.T., et al. (2020). Strain-tunable electronic and optical properties of monolayer GeSe: Promising for photocatalytic water splitting applications. Chemical Physics, 529, 110543.

15. Jappor, H.R., et al. (2019). Engineering the optical and electronic properties of Janus monolayer Ga2SSe by biaxial strain. Superlattices and 
Microstructures, 130, 545-553.

16. Obeid, M.M. (2020). Tuning the electronic and optical properties of Type-I PbI $2 / \alpha$-tellurene van der Waals heterostructure via biaxial strain and external electric field. Applied Surface Science, 508, 144824 .

17. Hoat, D.M., et al. (2019). Tuning the electronic structure of 2D materials by strain and external electric field: Case of GeI2 monolayer. Chemical Physics, 527, 110499.

18. Topsakal, M., et al. (2009). First-principles study of zinc oxide honeycomb structures. Physical Review B, 2009. 80(23): p. 235119.

19. Wu, P., et al., First-principles study of B, C, N and F doped graphene-like $\mathrm{MgO}$ monolayer. Physica E: Low-dimensional Systems and Nanostructures, 81, 713.

20. Naseri, M., et al. (2020). Electronic structure, optical and thermoelectric properties of cadmium chalcogenides monolayers. Optik, 210, 164567.

21. Zheng, H., et al. (2015). Monolayer II-VI semiconductors: A first-principles prediction. Physical Review B, 92(11), 115307.

22. Yandong, M., Dai, Y., Huang, B., (2011). Magnetism in non-transition-metal doped CdS studied by density functional theory. Computational Materials Science, 50(5), 1661-1666.

23. Akın, S., et al. (2013). Improvement of physical properties of $\mathrm{CdO}$ thin films by $\mathrm{Au}-\mathrm{Ag}$ nanocluster co-doping. Journal of Alloys and Compounds, 579, 272-278.

24. Kaviyarasu, K., et al. (2014). One dimensional wellaligned $\mathrm{CdO}$ nanocrystal by solvothermal method. Journal of Alloys and Compounds, 593, 67-70.

25. Tadjarodi, A., Imani, M., Kerdari, H. (2013). Application of a facile solid-state process to synthesize the $\mathrm{CdO}$ spherical nanoparticles. International Nano Letters, 3(1), 43.

26. Yufanyi, D., et al. (2014). CdO Nanoparticles by Thermal Decomposition of a CadmiumHexamethylenetetramine Complex. Journal of Materials Science Research, doi: 10.5539/ jmsr.v3n3p1.

27. Mohamed, S.H., et al. (2014). Synthesis, photoluminescence and optical constants evaluations of ultralong $\mathrm{CdO}$ nanowires prepared by vapor transport method. Journal of Alloys and Compounds, 609, 68-72.

28. Prakash, T., Neri, G., Kumar, E. R. (2015). A comparative study of the synthesis of $\mathrm{CdO}$ nanoplatelets by an albumen-assisted isothermal evaporation method. Journal of Alloys and Compounds, 624, 258-265.

29. Manickathai, K., Viswanathan, S.K., Alagar, M. (2008). Synthesis and characterization of $\mathrm{CdO}$ and CdS nanoparticles. Indian Journal of Pure $\mathcal{E}$ Applied Physics, 46, 561-564.

30. Tabatabaee, M., et al. (2013). A Simple Method for Synthesis of Cadmium Oxide Nanoparticles Using Polyethylene Glycol. Bulgarian Chemical Communications, 45, 90-92.

31. Vasheghani Farahani, S.K., et al. (2013). Temperature dependence of the direct bandgap and transport properties of CdO. Applied Physics Letters, 102(2), 022102

32. Al-Hada, N.M., et al. (2014). A facile thermaltreatment route to synthesize the semiconductor $\mathrm{CdO}$ nanoparticles and effect of calcination. Materials Science in Semiconductor Processing, 26, 460-466.

33. Aldwayyan, A.S., et al. (2013). Synthesis and Characterization of CdO Nanoparticles Starting from Organometalic Dmphen - CdI2 complex. International Journal of Electrochemicalscience, 8, $10506-10514$

34. Chaurasiya, R., et al. (2019). Strain Modulated Optoelectronic Properties of CdO Monolayer. Journal of Electronic Materials, 48(6), 3963-3969.

35. Chandiramouli, R., Jeyaprakash, B. G. (2013). Review of CdO thin films. Solid State Sciences, 16, 102-110.

36. Chaurasiya, R., Dixit, A. (2019). Point defects induced magnetism in $\mathrm{CdO}$ monolayer: A theoretical study. Journal of Magnetism and Magnetic Materials, 469 279-288.

37. Lu, H.B., et al. (2008). Fabrication of CdO nanotubes via simple thermal evaporation. Materials Letters, 62(24), 3928-3930.

38. Ma, X., J. Hu, Pan, B. (2017). Invariant wide bandgaps in honeycomb monolayer and singlewalled nanotubes of IIB-VI semiconductors. Nanotechnology, 28(35), 355201.

39. Zhi, Y., et al. (2018). Enhanced photocatalytic performance of $\mathrm{CdO} / \mathrm{g}-\mathrm{C} 6 \mathrm{~N} 6$ heterostructure. Materials Research Express, 6(3), 035910.

40. Khan, S.A., et al. (2015). Electronic structure and optical properties of $\mathrm{CdO}$ from bulk to nanosheet: DFT approach. Optical Materials, 47, 372-378.

41. Perdew, J.P., et al. (2008). Restoring the DensityGradient Expansion for Exchange in Solids and Surfaces. Physical Review Letters, 100(13), 136406.

42. Perdew, J.P., Burke, K., Ernzerhof, M. (1996). 
Generalized Gradient Approximation Made Simple. Physical Review Letters, 77(18), 3865-3868.

43. Dongho Nguimdo, G.M., Joubert, D.P. (2015). A density functional (PBE, PBEsol, HSE06) study of the structural, electronic and optical properties of the ternary compounds AgAlX2 $(\mathrm{X}=\mathrm{S}$, Se, Te). The European Physical Journal B, 88(5), 113.

44. Bolívar Marín, Y., Alcalá Varilla, L.A., González Ramirez, I.A. (2019) Comparative study of the effect of the exchange-correlation functional on the structural and electronic properties of rutile. Journal of Physics: Conference Series, 1386, 444.
45. Kresse, G., Furthmüller, J. (1996). Efficient iterative schemes for ab initio total-energy calculations using a plane-wave basis set. Physical Review B, 54 (16), 11169-11186.

46. Blöchl, P.E. (1994). Projector augmented-wave method. Physical Review B, 50(24), 17953-17979.

47. Monkhorst, H.J., Pack, J.D. (1976). Special points for Brillouin-zone integrations. Physical Review B, 13(12), 5188-5192. 